\title{
Incidental thyroid 99mTc-MDP uptake in a patient affected by differentiated thyroid cancer
}

\author{
Domenico Albano', Gian Carlo Magri', Giorgio Treglia², Francesco Bertagna ${ }^{1}$ \\ ${ }^{1}$ Nuclear Medicine, University of Brescia and Spedali Civili Brescia, Brescia, Italy \\ 2Department of Nuclear Medicine and PET/CT Center, Oncology Institute of Southern Switzerland, Bellinzona, Switzerland
}

[Received 21 I 2015; Accepted 18 VIII 2016]

\begin{abstract}
We report a case of incidental uptake of ${ }^{99 \mathrm{~m} T c-m e t h y l e n e ~ d i p h o s p h o n a t e ~ i n ~ t h e ~ t h y r o i d ~ i n ~ a ~ 62-y e a r-o l d ~ f e m a l e ~ w i t h ~ a ~ h i s t o r y ~}$ of breast cancer treated with quadrantectomy, chemotherapy and radiotherapy, who underwent total-body-bone scintigraphy during follow up. Planar scintigraphy was followed by neck SPECT-CT that demonstrated an area of increased tracer uptake in the neck at the left lobe of the thyroid. Neck ultrasonography showed a nodule corresponding to SPECT-CT finding and the ultrasound-guided fine needle aspiration documented the presence of papillary carcinoma. The patient underwent total thyroidectomy and ipsilateral lymphadenectomy; histological examination confirmed the presence of a papillary carcinoma and the patient underwent ablation therapy with iodine-131.
\end{abstract}

KEY words: incidental, ${ }^{99 m}$ Tc-MDP, bone scintigraphy, differentiated thyroid carcinoma

Nucl Med Rev 2016; 19, suppl. B: B8-B10

\section{Background}

${ }^{99 m} T c-M D P$ bone scintigraphy is a nuclear scanning test to find certain abnormalities in bone. It is mainly used to help diagnose a number of conditions related to bones, including primary bony malignancies, metastatic diseases that have spread to the bone, occult fractures and other conditions. In addition, this examination is often used for the follow up or response to treatment of some solid cancers like prostate and breast cancers [1]. Besides, the bone scan delineates a wide spectrum of non-osseous disorders: neoplastic, hormonal, inflammatory, ischemic, traumatic, excretory and artifactual entities may demonstrate abnormal soft tissue uptake of ${ }^{99 \mathrm{mTc}-M D P}$ [2]

\section{Case report}

A 62-years-old female with a history of breast cancer (invasive ductal carcinoma) previously treated with quadrantectomy with axillary dissection, chemotherapy and radiotherapy underwent ${ }^{99 m}$ Tc-methylene diphosphonate (MDP) bone scintigraphy during the follow-up in order to verify the presence of skeletal metastases.

Correspondence to: Domenico Albano, MD Nuclear Medicine, Spedali Civili di Brescia

P.le Spedali Civili, 1, 25123 Brescia, Italy

Tel.: 39303995 468; Fax: + 39303995420

E-mail: doalba87@libero.it
The study was negative for bone metastases but revealed an area of focal tracer uptake at the base of the neck more visible in the anterior view (Figure 1-A, 1-B), corresponding to the left lobe of the thyroid gland at the subsequent single-photon emission computed tomography-computed tomography (SPECT-CT) acquisition of the neck and thorax (Figure 1-C). CT images revealed a $2 \mathrm{~cm}$ solid roundish nodule. The neck ultrasonography showed a solid nodule in the left lobe of the thyroid, almost entirely calcified mainly in the capsule with a diameter of $2 \mathrm{~cm}$. Ultrasound-guided fine needle aspiration (FNA) was positive for papillary carcinoma (TIR6 Bethesda system). The patient underwent total thyroidectomy associated with ispilateral lymphadenectomy. Histological examination of the primary tumor revealed a conventional papillary carcinoma with thyroid capsule invasion and extrathyroidal extension with two left lateral cervical metastatic nodes (pT3N1a AJCC 2010 classification). Therefore, after the withdrawal of thyroid hormone therapy, she underwent iodine-131 (I131) therapy (3.7 GBq) for remnant ablation. After 72 hours the 1131 total body planar scintigraphy (Figure 2-A) and the subsequent SPECT/CT of neck and thorax (Figure 2-B1-B3) showed high focal uptake of tracer in the upper central area of the neck due to thyroglossal duct remnant and slight uptake in left thyroid bed corresponding to residual thyroid tissue.

\section{Discussion}

In literature many cases of incidental uptakes of ${ }^{99 \mathrm{~m}} \mathrm{Tc}-\mathrm{MDP}$ at extraosseous sites have been reported: neoplastic, hormonal, 
1-A
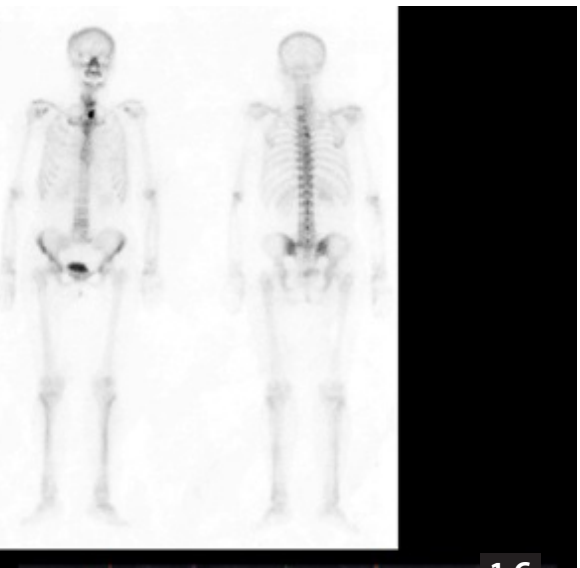

$1-\mathrm{C}$

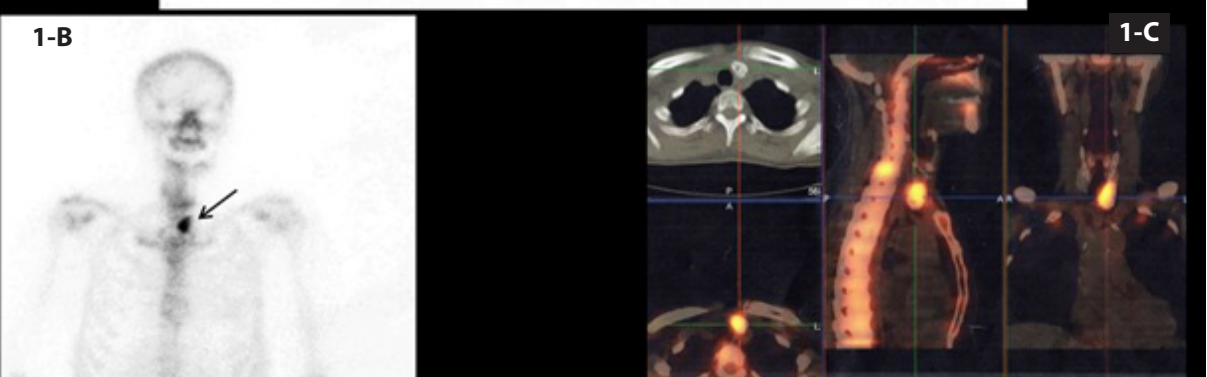

Figure 1A-C. In this figure ${ }^{99 \mathrm{~m} T \mathrm{Tc}-m e t h y l e n e ~ d i p h o s p h o n a t e ~(M D P) ~ b o n e ~ s c i n t i g r a p h y ~ i s ~ s h o w e d ; ~}$

1-A. Anterior and posterior views of the study showing the lesion (black arrow); 1-B. An enlargement of the previous study to show more clearly the lesion in the neck; $1-\mathbf{C}$. An acquisition of the neck and thorax of single-photon emission computed tomography-computed tomography (SPECT-CT)

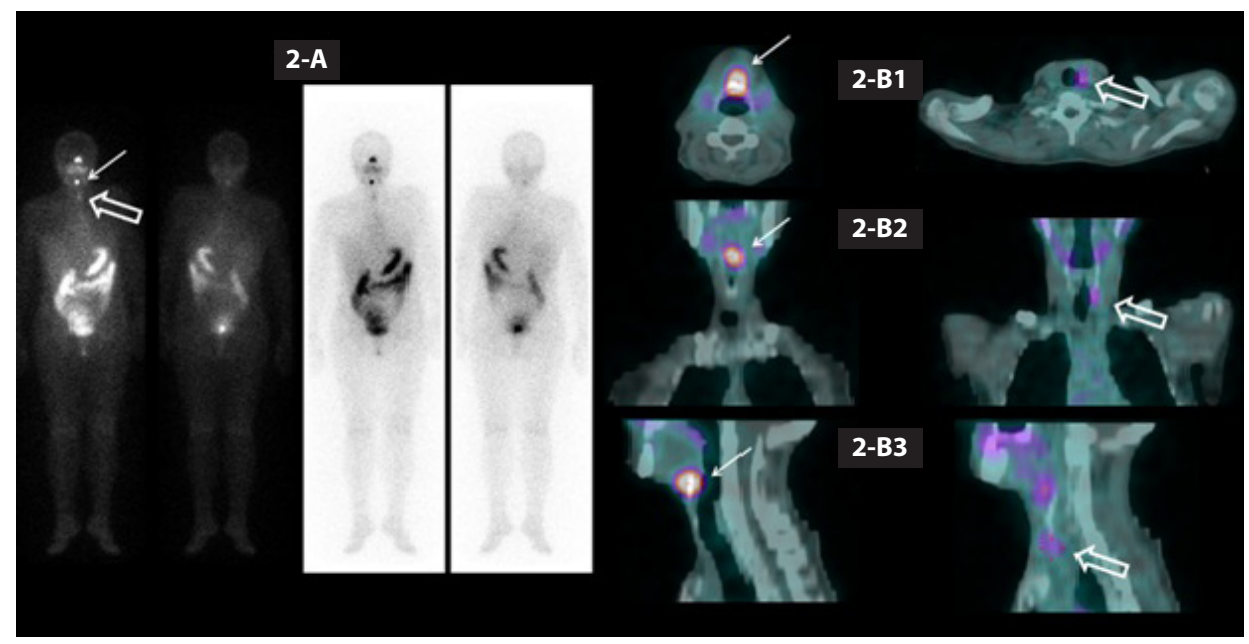

Figure 2-A. Anterior and posterior view of $\mathrm{I}^{131}$ total body scintigraphy performed in the patient after radiometabolic therapy that showed focal uptake of tracer in the upper central area of the neck due to thyroglossal duct remnant (slim arrow) and slight uptake in left thyroid bed corresponding to residual parenchymal (empty arrow); 2-B1-B3. Axial, coronal and sagittal I131 SPECT-TC fused images

inflammatory, ischemic, traumatic, excretory and artifactual entities may demonstrate abnormal soft tissue uptake of ${ }^{99 m}$ Tc-MDP [2] .

Radionuclide imaging with ${ }^{99 m} \mathrm{Tc}-\mathrm{MDP}$ generally depends on the uptake of the tracer by normal osteoblasts reacting to bone damage produced by malignant lesions. However in certain disorders, especially those characterized by soft tissue calcification, the radiopharmaceutical may accumulate within pathologic tissue itself. ${ }^{99 m}$ Tc-MDP thyroid incidental uptake is not very frequent and it is possibly caused by the presence of calcification $[3,4]$, biopsy procedure [5], anaplastic carcinoma [6] or metastatic calcifications in patients affected by primary hyperparathyroidism [7]. It can also happen to have focal tracer uptake in the thyroid autonomous nodules that is probably caused by dissociation of ${ }^{99 \mathrm{~m} T c}$ from MDP. Calcified nodules may show high uptake of this radiopharmaceutical. Calcification within the thyroid may happen in both benign and malignant diseases [8]. It is well known 
that microcalcifications are associated with thyroid malignancy [9]; instead the association between macrocalcifications and thyroid cancer remains still under discussion but it is mainly related to benign disease $[10,11]$.

The peculiarity of this report is that the finding of a nodule entirely calcified is associated with malignancy, although in clinical practice it is usually associated with benign disease. In conclusion, this report underlines the importance of further investigation of incidental findings in the thyroid at ${ }^{99 m} \mathrm{Tc}-\mathrm{MDP}$ bone scan, potentially of clinical relevance and pivotal for an adequate patient management.

\section{References}

1. Bares R. Skeletal scintigraphy in breast cancer management. QJ Nucl Med 1998; 42: 43-48

2. Peller P, Ho V, Kransdorf M. Extraosseous Tc-99m MDP uptake: a pathophysiologic approach. Radiographics 1993; 13: 715-734.

3. Kim YC. Thyroid uptake on bone scan in a large, multinodular, nontoxic goiter with calcification degeneration. Clin Nucl Med 1980; 5: 561-562.
4. Bertagna F, Bosio G, Giubbini R. Incidental thyroid Tc-99m methylene diphosphonate (MDP) uptake in a patient affected by polynodular goiter at bone scintigraphy. Nucl Med Rev Cent East Eur 2009; 12: 81-82.

5. Rehm PK, Sharma S. Focal thyroid uptake on bone scan due to thyroid biopsy. Clin Nucl Med 2004; 29: 849-851.

6. Montes TC, Munoz C, Rivero Jl et al. Uptake of TC-99m sestamibi and Tc-99m MDP in anaplastic carcinoma of the thyroid. Clin Nucl Med 1999; 24: 355-356.

7. Amico S, Lucas P, Diebold MD et al. Metastatic calcification in the thyroid gland demonstrated on bone scan in a patient with primary hyperparathyroidism. J Nucl Med 1986; 2: 373-376.

8. Khoo ML, Asa SL, Witterick IJ, Freeman JL. Thyroid calcification and its association with thyroid carcinoma. Head Neck 2002; 24: 651-655.

9. Kim BK, Choi YS, Kwon HJ et al. Relationship between patterns of calcification in thyroid nodules and histopathologic findings. Endocrine J 2013; 60: $155-160$

10. Lee J, Lee SY, Cha SH et al. Fine-needle aspiration of thyroid nodules with macrocalcification. Thyroid 2013; 23: 1106-1112.

11. Park YJ, Kim JA, Son EJ et al. Thyroid nodules with macrocalcification: sonographic findings predictive of malignancy. Yonsei Med J 2014; 55: 339-344. 